\title{
Numerical investigation of thermomagnetic convection of electrically conducting fluids under an inclined magnetic field using lattice Boltzmann method

\author{
Nan Xie ${ }^{a}$, Changwei Jiang ${ }^{b, ~}$, Yihai $\mathrm{He}^{c}$, Ming $\mathrm{Yao}^{\mathrm{d}}$
}

\begin{abstract}
Key Laboratory of Efficient and Clean Energy Utilization of College of Hunan Province, School of Energy and Power Engineering, Changsha University of Science and Technology, Changsha 410114, China
\end{abstract}

aemail: xienanpi@163.com, ${ }^{\mathrm{b} e m a i l: c w \_j i a n g @ 163 . c o m ~}$

Keywords: lattice Boltzmann method; numerical simulation; thermomagnetic convection; inclined magnetic field; electrically conducting fluid

\begin{abstract}
In the present work, natural convection in a square cavity filled with an electrically conducting fluid has been numerically investigated in the presence of an inclined magnetic field using lattice Boltzmann method. The vertical walls of this two-dimensional cavity are heated differentially while the horizontal walls are assumed to be adiabatic. The flow and temperature field distributions are obtained and the average Nusselt numbers on the left hot wall is calculated. Available results reveal that both the Hartmann number and the inclination angles of magnetic field have significant influence on the heat transfer.
\end{abstract}

\section{Introduction}

Convective heat transfer in the presence of magnetic field comes to be a research focus nowadays because of its wide reach in engineering practices, and applying magnetic field to strengthen or control heat transfer properties in the cavity gains its popularity among a large quantity of researchers.Investigators have already proved that the Rayleigh number has a positive effect on the heat transfer while the Hartmann number has a negative one[1-4]. Published literature [1-10] are based on the traditional Computational Fluid Dynamics methods such as the finite volume method, the finite difference method and so on, while the lattice Boltzmann method has already become an available and brand new numerical method after the theoretical breakthrough and research progress for more than 20 years. LBM is a programming easily numerical method with simple algorithms which also has capabilities of simulation for microflows, nanopaticles, crystal growth, porous media and many other complex convective heat transfer problems which traditional methods cannot achieve. So it is of great necessity to investigate the problem in an inclined magnetic field utilizing lattice Boltzmann method. In this study, standard two-dimensional model with nine velocities and temperature-density double distribution equations of LBM are employed to numerically investigate the thermomagnetic convection of an electrically conducting fluid in square cavity under an inclined magnetic field.

\section{Problem statement}

The geometry of present problem are clearly described in Fig.1, which consists of a two-dimensional square cavity with a height of L, and the cavity is placed horizontally. Vertical walls of this cavity are heated differentially, the temperature of left wall is maintained at Th while the temperature of right wall is fixed at Tc, and Th is hotter than Tc. The horizontal walls are assumed to be insulated. The inclination angle of inclined uniform magnetic field is $\theta$. Cartesian coordinates are utilized and the origin of coordinates is fixed at the left bottom corner. The enclosure is filled with an electrically conducting fluids with a Prandtl number of 0.025 . 


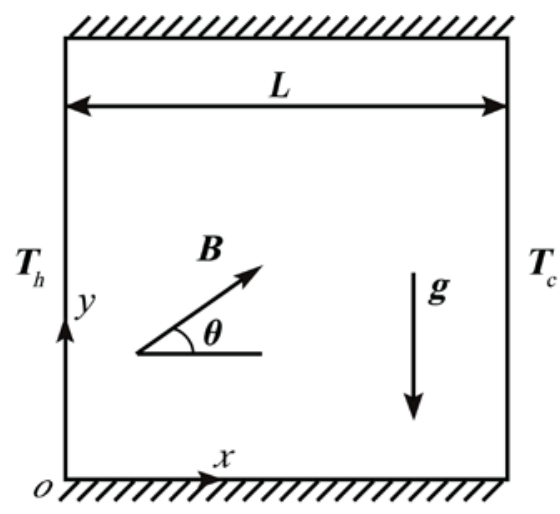

Fig.1 Physical model and Cartesian coordinates.

\section{Governing equations}

The electrically conducting fluid is assumed to be Newtonian and incompressible. The flow in this enclosure is steady and there is also no phase change, and all the effects of Joule heating, induced magnetic field and viscous dissipation on natural convection are neglected. Accordingly, the dimensionless governing equations can be described as[8]:

$$
\begin{aligned}
\frac{\partial U}{\partial X}+\frac{\partial V}{\partial Y} & =0 \\
U \frac{\partial U}{\partial X}+V \frac{\partial U}{\partial Y} & =-\frac{\partial P}{\partial X}+\operatorname{Pr}\left(\frac{\partial^{2} U}{\partial X^{2}}+\frac{\partial^{2} U}{\partial Y^{2}}-H a^{2} U \sin ^{2} \theta+H a^{2} V \sin \theta \cos \theta\right) \\
U \frac{\partial V}{\partial X}+V \frac{\partial V}{\partial Y} & =-\frac{\partial P}{\partial Y}+\operatorname{RaPr\Theta }+\operatorname{Pr}\left(\frac{\partial^{2} V}{\partial X^{2}}+\frac{\partial^{2} V}{\partial Y^{2}}-H a^{2} V \cos ^{2} \theta+H a^{2} U \sin \theta \cos \theta\right) \\
U \frac{\partial \Theta}{\partial X}+V \frac{\partial \Theta}{\partial Y} & =\frac{\partial^{2} \Theta}{\partial X^{2}}+\frac{\partial^{2} \Theta}{\partial Y^{2}}
\end{aligned}
$$

Rayleigh number, Prandtl number and Hartmann number are defined as:

$$
R a=\frac{g \beta\left(T_{h}-T_{c}\right) L^{3}}{v \alpha}, \quad \operatorname{Pr}=\frac{v}{\alpha}, H a=\sqrt{\frac{\sigma B^{2} L^{2}}{v \rho}}
$$

Where the $\mathrm{X}$ and $\mathrm{Y}$ are the dimensionless coordinates. $\mathrm{U}$ and $\mathrm{V}$ are the dimensionless velocities. $\Theta$ is the dimensionless temperature. $P$ is the dimensionless pressure. T0, Th and Tc are the reference temperature and steady temperature on the vertical walls. $\mathrm{L}$ is the length of square cavity. $\mathrm{B}$ is the magnitude of uniform magnetic field. $\mu$ is the dynamic viscosity. $v$ is the kinematic viscosity. $\theta$ is the angle of inclined magnetic field with respect to the horizontal plane. $g$ is the gravity acceleration. $\beta$ is the thermal expansion coefficient. $\sigma$ is the conductivity of fluid. $\rho$ is the fluid density. $\alpha$ is the thermal diffusivity. T0 is the reference temperature.

\section{Lattice Boltzmann equations}

In present research, standard D2Q9 model of LBM is employed for flow and temperature in this two-dimensional cavity. The discrete velocity set for the D2Q9 model is:

$$
e_{k}=\left\{\begin{array}{clrl}
(0,0), & k & =0 \\
c\left(\cos \left[(k-1) \frac{\pi}{2}\right], \sin \left[(k-1) \frac{\pi}{2}\right]\right), & k & =1,2,3,4 \\
\sqrt{2} c\left(\cos \left[(2 k-1) \frac{\pi}{4}\right], \sin \left[(2 k-1) \frac{\pi}{4}\right]\right), & k=5,6,7,8
\end{array}\right.
$$

Where $e_{k}$ are the discrete velocities of lattice. The weighted factors of lattice $\omega_{\mathrm{k}}$ are assigned as: $\omega_{0}=0, \omega_{1-4}=1 / 9, \omega_{5-8}=1 / 36$. The lattice Boltzmann equations of the flow and temperature field with external forces can be written as follows:

$$
\begin{aligned}
& f_{k}(x+\Delta x, t+\Delta t)=f_{k}(x, t)\left[1-\omega_{m}\right]+\omega_{m} f_{k}^{e q}(x, t)+F_{k} \\
& g_{k}(x+\Delta x, t+\Delta t)=g_{k}(x, t)\left[1-\omega_{s}\right]+\omega_{s} g_{k}^{e q}(x, t)
\end{aligned}
$$

Here $f$ is the density distribution functions, and $g$ is the internal energy distribution functions. The 
external force term $F_{k}$, factors $\omega_{m}$ and $\omega_{s}$ are defined as:

$F_{k}=3 \cdot \omega_{k} \cdot\left(\frac{H a^{2} \mu}{L^{2}}\left(v \cos \theta \sin \theta-u \sin ^{2} \theta\right)+g \beta \rho\left(T-T_{0}\right)+\frac{H a^{2} \mu}{L^{2}}\left(u \cos \theta \sin \theta-v \cos ^{2} \theta\right)\right)$

$\omega_{m}=\frac{1}{3 \cdot v+0.5}, \quad \omega_{s}=\frac{1}{3 \cdot \alpha+0.5}$

And the equilibrium density distribution functions $f^{e q}$ and the equilibrium internal energy distribution functions $g^{e q}$ can be defined as:

$$
\begin{aligned}
& f_{k}^{e q}=\omega_{k} \rho\left[1+3 \frac{\left(e_{k} \cdot u\right)}{c^{2}}+4.5 \frac{\left(e_{k} \cdot u\right)^{2}}{c^{4}}-1.5 \frac{u^{2}}{c^{2}}\right] \\
& g_{k}^{e q}=\omega_{k} T\left[1+3 \frac{\left(e_{k} \cdot u\right)}{c^{2}}\right]
\end{aligned}
$$

At last, the macroscopic variables can be calculated by the following formula:

$$
\rho(x, t)=\sum_{k} f_{k}(x, t), \quad \rho u(x, t)=\sum_{k} f_{k}(x, t) e_{k}+F_{k}, T=\sum_{k} g_{k}(x, t)
$$

\section{Description of numerical results}

The average Nusselt number on hot wall is used in this work to describe the heat transfer properties:

$$
N u_{\mathrm{m}}=\int_{0}^{1}-\left.\frac{\partial \Theta}{\partial X}\right|_{X=0} d Y
$$

\section{Results and discussion}

Fig.2 depicts the effect of Hartmann number on the streamlines at $\mathrm{Ra}=104$ and 105. It describes the suppression on the convective heat transfer by the applied magnetic field. The stream function on the central streamline decreases significantly with the increasing Hartmann number which indicates that both the intensity of flow and the heat transfer rate are weakening. The central region of streamlines diminishes gradually with the growing Hartmann number and breaks into two sections of extreme value on the upper and lower end of vertical center line eventually. Contours that nearly parallel to the vertical walls are spotted when the magnetic field is strong enough which declares the dominant role of heat conduction in the heat transfer .

(a)

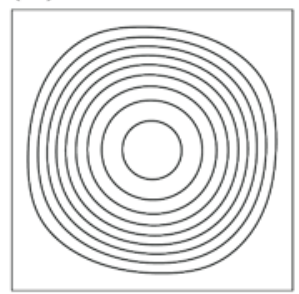

(e)

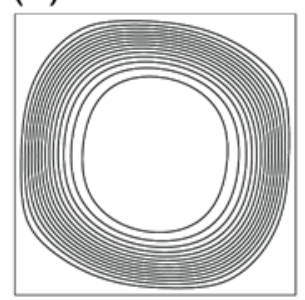

(b)

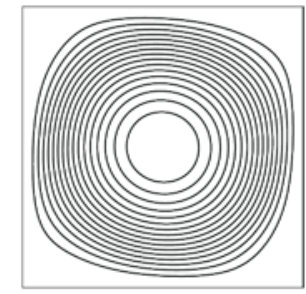

(f)

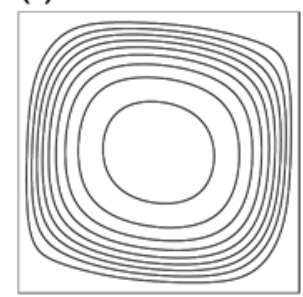

(c)

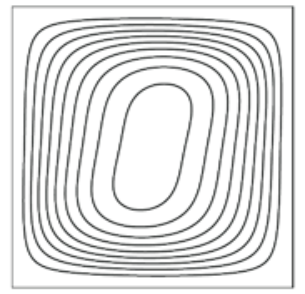

(g)

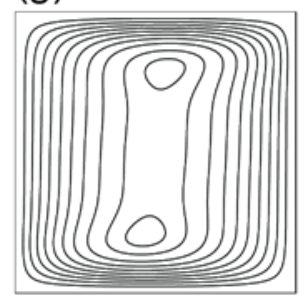

(d)

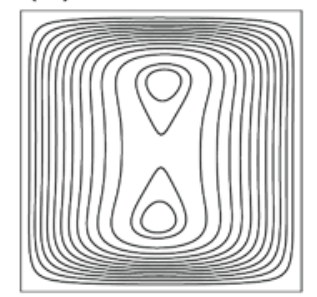

(h)

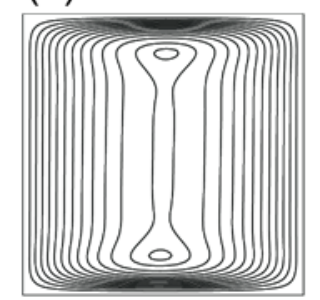

Fig.2 Streamlines under horizontal magnetic field: (a) $\mathrm{Ra}=10^{4}, \mathrm{Ha}=0$, (b) $\mathrm{Ra}=10^{4}, \mathrm{Ha}=10$, (c) $\mathrm{Ra}=10^{4}$, $\mathrm{Ha}=50,(\mathrm{~d}) \mathrm{Ra}=10^{4}, \mathrm{Ha}=100,(\mathrm{e}) \mathrm{Ra}=10^{5}, \mathrm{Ha}=0$, (f)Ra=10 ${ }^{5}, \mathrm{Ha}=30,(\mathrm{~g}) \mathrm{Ra}=10^{5}, \mathrm{Ha}=160$, (h) $\mathrm{Ra}=10^{5}$, $\mathrm{Ha}=320$. 

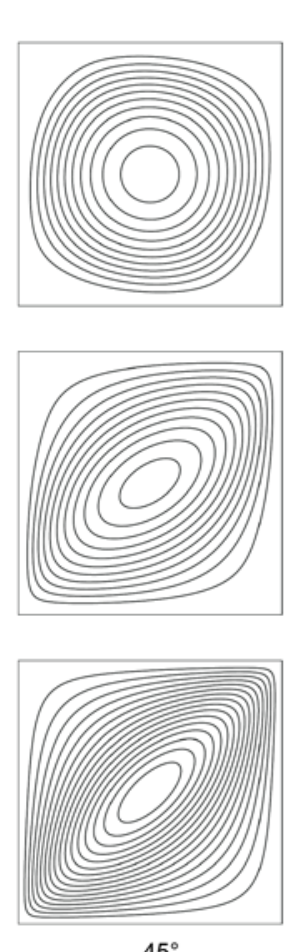

$45^{\circ}$

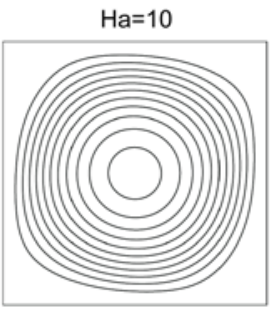

$\mathrm{Ha}=50$

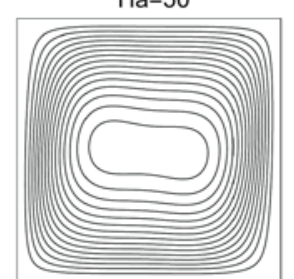

$\mathrm{Ha}=100$

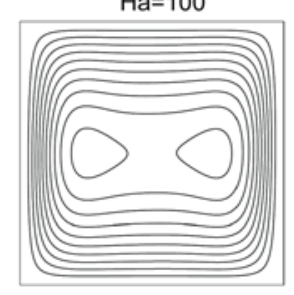

$90^{\circ}$
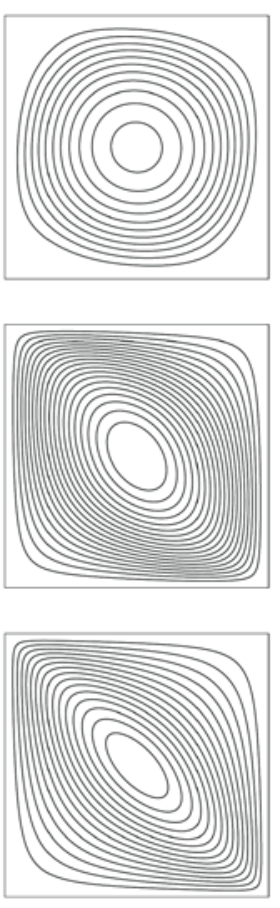

$135^{\circ}$

Fig.3 Streamlines of the fluid with a Prandtl number of 0.025 under inclined magnetic field at $\mathrm{Ra}=10^{4}$

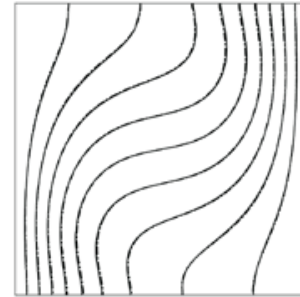

$\mathrm{Ha}=10$

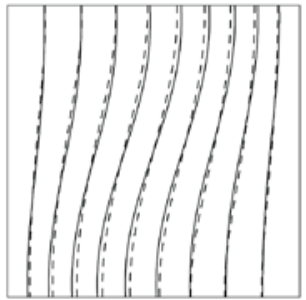

$\mathrm{Ha}=50$

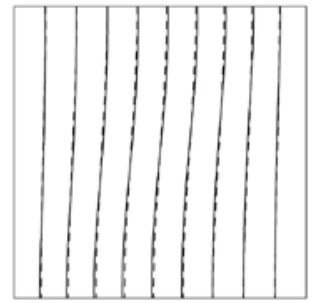

$\mathrm{Ha}=100$

Fig.4 Isotherms in the inclined magnetic field: $45^{\circ}$ (solid), $90^{\circ}$ (dashed), $135^{\circ}$ (dotted).

Streamlines in the enclosure under inclined magnetic field are shown in Fig.3. The square cavity is placed horizontally with inclination angles varying from $45^{\circ}, 90^{\circ}$ to $135^{\circ}$ at $\mathrm{Ra}=104$, and the set value of Ha are 10, 50 and 100. It is found that the increase of Hartmann number significantly augments the effect of inclination angles on the streamlines and symmetrical contours are obtained when tilting the magnetic field about $45^{\circ}$ and $135^{\circ}$. This vertical symmetry is caused by the combined influence of gravity, buoyancy lift and the inclined magnetic field etc. When the uniform magnetic field is tilted about $90^{\circ}$, the center streamline stretches horizontally and divides into two sections when the magnetic field is powerful. Fig.4 describes the effect of inclination angles on the isotherms and it can be observed that the effect is insignificant.

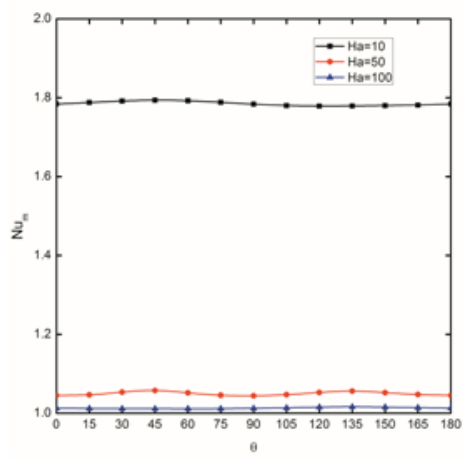

Fig.5 Effect of inclined angle on average Nusselt numbers for different Hartmann numbers.

Fig. 5 depicts the variation of average Nusselt number with the changing inclination angles for 
various Hartmann numbers at $\mathrm{Ra}=104$. Only one obvious peak value of average Nusselt number can be observed at $\mathrm{Ha}=10$ while at $\mathrm{Ha}=50$, there exist two peak values at $45^{\circ}$ and $135^{\circ}$ in the same period as a consequence of the increasing magnetic field. At $\mathrm{Ha}=100$, there is no obvious change of the average Nusselt number which indicates the effect of inclination angles is insignificant when the magnetic field is strong.

\section{Conclusions}

In this paper, thermomagnetic convection in a square cavity with differentially heated vertical walls and adiabatic horizontal walls is numerically investigated utilizing lattice Boltzmann method under an inclined magnetic field. The double distribution equations and D2Q9 model of LBM are employed to analyze the heat transfer characteristics. Relevant parameters are: the Rayleigh number 104, Hartmann number from 0 to 100 with intervals of 10, inclination angles of the magnetic field varying from $0^{\circ}$ to $180^{\circ}$ with intervals of $15^{\circ}$. Comparisons of the flow, temperature distributions and heat transfer properties are illustrated clearly and conclusions are summarized as follows:

a) Numerical investigation in present research proves the validity and correctness of the lattice Boltzmann method in handling the thermomagnetic convection of electrically conducting fluids in a square cavity.

b) The average Nusselt number decreases obviously with the increasing Hartmann number. Therefore, augment of the magnetic field strength will tremendously suppresses the flow intensity and the heat transfer rate.

c) The inclination angles obviously effects the average Nusselt number at low Hartmann number. In the weak magnetic field, its effect on the flow structure and heat transfer is remarkable.

\section{References}

[1] B. Ghasemi, S.M. Aminossadati, A. Raisi. Magnetic field effect on natural convection in a nanofluid-filled square enclosure[J]. International Journal of Thermal Sciences. 2011(50): 1748-1756.

[2] Ali Meerali Jasim Al-Zamily. Effect of magnetic field on natural convection in a nanofluid-filled semi-circular enclosure with heat flux source[J]. Computers \& Fluids. 2014(103): 71-85.

[3] M. Ashouri, B. Ebrahimi, M.B. Shafii, M.H. Saidi, M.S. Saidi. Correlation for Nusselt number in pure magnetic convection ferrofluid flow in a square cavity by a numerical investigation[J]. Journal of Magnetism and Magnetic Materials. 2010(322): 3607-3613.

[4] Mohsen Pirmohammadi, Majid Ghassemi. Effect of magnetic field on convection heat transfer inside a tilted square enclosure[J]. International Communications in Heat and Mass Transfer. 2009(36): 776-780.

[5] M. Sankar, M. Venkatachalappa, I.S. Shivakumara. Effect of magnetic field on natural convection in a vertical cylindrical annulus[J]. International Journal of Engineering Science. 2006(44): 1556-1570.

[6] M. Sankar, M. Venkatachalappa, Younghae Do. Effect of magnetic field on the buoyancy and thermocapillary driven convection of an electrically conducting fluid in an annular enclosure[J]. International Journal of Heat and Fluid Flow. 2011(32): 402-412.

[7] Jenn-Shyang Kuo, Jik Chang Leong. Analysis of a conducting fluid in a thin annulus with rotating insulated walls under radial magnetic effect[J]. Applied Mathematical Modelling. 2013(37): 3021-3035.

[8] P.X. Yu, J.X. Qiu, Q. Qin, Zhen F. Tian. Numerical investigation of natural convection in a rectangular cavity under different directions of uniform magnetic field[J]. International Journal of Heat and Mass Transfer. 2013(67): 1131-1144.

[9] M. Sathiyamoorthy, Ali Chamkha. Effect of magnetic field on natural convection flow in a liquid gallium filled square cavity for linearly heated side wall(s)[J]. International Journal of Thermal Sciences. 2010(49): 1856-1865. 
[10]Hillal M. Elshehabey, F.M. Hady, Sameh E. Ahmed, R.A. Mohamed. Numerical investigation for natural convection of a nanofluid in an inclined L-shaped cavity in the presence of an inclined magnetic field[J]. International Communications in Heat and Mass Transfer. 2014(57): 228-238. 